American Journal of Applied Sciences 8 (11): 1149-1153, 2011

ISSN 1546-9239

(C) 2011 Science Publications

\title{
Specification of Dynamic Time Series Model with Volatile-Outlier Input Series
}

\author{
${ }^{1}$ T.A. Lasisi, ${ }^{1}$ D.K. Shangodoyin, ${ }^{2}$ O.O. Sangodoyin, \\ ${ }^{1}$ W.M. Thupeng and ${ }^{1}$ P.M. Kgosi \\ ${ }^{1}$ Faculty of Social Sciences, \\ ${ }^{2}$ Faculty of Business, \\ University of Botswana, Botswana
}

\begin{abstract}
Problem statement: This study considers the precision of the output series generated from aberrant input series in the context of the distribution of the dynamic estimate and also investigate relative merit of analyzing residuals with outliers for a volatility input dynamic model. Approach: The study developed a methodology for checking volatility at every time point and evaluates the influence of volatility and outliers on both the estimates of the fitted Dynamic Model (DM) and test criterion for model adequacy. Results: Both the analytical and empirical findings in this study reveal that outliers affect significantly the estimates of the dynamic model and there was a masking effect of volatility with outliers in the series and therefore jeopardizes test criterion for model adequacy because outlier series were embedded in its computation. Conclusion: The analysis of outlier in dynamic model specification can involve the determination of volatility, most especially in economic series for which causal relationship can proffer some evidence based solutions to decision makers on pressing economic issues. The model specified in this study has shown the influence of outlier embedded with volatility in empirical study on dynamic function modelling. In the first instance, outlier significantly affects the estimates of the model, apart from this; the model residual is affected, these have a combine effect on the precision of output generated.
\end{abstract}

Key words: Dynamic model, volatility outliers, model specification, model analyzing residuals, outlier series, series generated, outlier contaminated, standard deviation, outlying observation

\section{INTRODUCTION}

The problem of fitting dynamic model in time series analysis is one challenge of testing model specification, especially when there is need for measuring the precision of the generated output series based on the inference about the parameters, as well as test of adequacy of fitted model is the presence of outlier and volatile input series. Sarnaglia et al. (2010) have emphasized that outlying observation may be strong enough to ruin any kind of traditional methods of estimation and much effort in time series analysis is geared towards overcoming or alleviating the problem of outlying observation in data analysis. Volatility in international commodity price usually emanate from two main sources, either through a change in world prices or fluctuation in exchange rate (George Rapsomanikis and Alexander, 2006); the state of the two major sources determine the eventual domestic trade price of a commodity over a period of time, hence the need for studying the behavior of volatility economic time series.

In this study, we consider the estimation of the dynamic parameter space and the analysis of the residuals of a dynamic model in the presence of outlier input series embedded with volatility.

\section{MATERIALS AND METHODS}

Specification of outliers based dynamic modelwith measure of volatility: Let the Dynamic Model (DM) of order $(\mathrm{p}, \mathrm{q})(\mathrm{DM}(\mathrm{p}, \mathrm{q}))$ be defined as:

$\delta(B) Y_{t}=\omega(B) X_{t}+\varepsilon_{t}$

Where:

$\delta(B)=\sum_{j=0}^{p} \delta_{j} B^{j}, \delta_{0}=1, \omega(B)=\sum_{j=0}^{q} \omega_{j} B^{j}$ and $\omega_{o}=1 . B$ is the backward shift operator defined as $\mathrm{B} \omega_{\mathrm{t}}=\omega_{\mathrm{t}-1}, \varepsilon_{\mathrm{t}}$ is the 
stochastic disturbance term at time $\mathrm{t}$ and there is no delay in the response of $\mathrm{Y}$ to a change in $\mathrm{X}$, this is assumed.

Suppose that the autocorrelation in $\varepsilon_{\mathrm{t}}$ is the characterized by an Auto-Regressive-Moving Average (ARMA) of order (r, s) defined as Eq. 2:

$$
\varepsilon_{\mathrm{t}}=\frac{\theta(\mathrm{B}) \mathrm{e}_{\mathrm{t}}}{\phi(\mathrm{B})}
$$

where, $\phi(B)=\sum_{j=0}^{r} \phi_{j} B^{j}, \theta(B)=\sum_{j=0}^{s} \theta_{j} B^{j}$ and $\phi_{0}=\theta_{0}=1 .\left\{e_{t}\right\}$ are the uncorrelated normal deviates with mean 0 and variance $\sigma_{\mathrm{e}}{ }^{2}$.

The combined dynamic disturbance model is then:

$$
\delta(B) Y_{t}=\omega(B) X_{t}+\frac{\theta(B) e_{t}}{\phi(B)}
$$

Now assume a gross error model on $X_{t}$, an outlier contaminated input series, we obtain Eq. 4:

$$
X_{t}=Z_{t}+D \xi_{t}^{T}
$$

where, $Z_{t}$ is an outlier free volatile series and the volatility in $\mathrm{Z}_{\mathrm{t}}$ is measured by $\mathrm{h}(\mathrm{p})=\sigma \sqrt{\mathrm{p}}$, where $\sigma$ is the standard deviation of the outlier free observations for the given period. $\mathrm{D}$ is the magnitude of the outlier at time $\mathrm{t}$ $=\mathrm{T}$ and $\xi_{\mathrm{t}}^{\mathrm{T}}=\left\{\begin{array}{l}=1 \text { when } \mathrm{t}=\mathrm{T} \\ =0 \text { when } \mathrm{t} \neq \mathrm{T}\end{array} . \mathrm{T}\right.$ is the timing occurrence of the outlier.

From Eq. 1 through (3), we derive the dynamic outlier model as Eq. 5:

$$
\delta(B) Y_{t}=\omega(B)\left[h_{t}(t)+D \xi_{t}^{T}\right]+e_{t}
$$

Given the fact that the distribution of dynamic parameter space does not influence the disturbance space (Shangodoyin, 2008), we have the outlier based dynamic structure as Eq. 6:

$$
\begin{aligned}
Y_{t} & =\frac{\omega(B) h_{t}(t)}{\delta(B)}+\frac{\omega(B) D \xi_{t}^{T}}{\delta(B)}+\delta^{-1}(B) e_{t} \\
& =\pi(B) h_{t}(t)+R_{t}
\end{aligned}
$$

The $R_{t}$ in the second part of Eq. 6 is the residual of the outlier induced model and for the model in Eq. 6, we make the following assumptions:

- All $X_{t}$ in (4) are suspected outlier, in other words, $\xi_{t}^{T}=1$ for all $\mathrm{t}=\mathrm{T}$ unless otherwise confirmed with the procedure for testing presence of outliers (Tsay, 1986)
- The function $h_{t}(t)$ is significantly volatile for all time $\mathrm{t}$

- The series $Y_{t}, D_{t}$ and $h_{t}(t)$ are stationary and mutually independent of $\varepsilon_{\mathrm{t}}$

- The errors $e_{t}$ are additive with respect to the influence of $Y_{t}, D_{t}$ and $h_{t}(t)$

- The errors $\left\{e_{t}\right\}$ are stationary, independent and identically distributed normal with mean 0 and finite variance $\sigma_{\mathrm{e}}^{2}$

The $\left\{Y_{t}\right\},\left\{X_{t}\right\}$ and $\left\{D_{T}\right\}$ are bounded and for fixed p, $q$ and $m$; the limits of the matrices $\lim _{n \rightarrow \infty} \frac{1}{n} \sum_{1}^{n} Y_{t-k} Y_{t-j}$, $\lim _{n \rightarrow \infty} \frac{1}{n} \sum_{1}^{n} X_{t-k} X_{t-j} \quad$ and $\lim _{n \rightarrow \infty} \frac{1}{n} \sum_{T=1}^{m} D_{T-k} D_{T-j} \quad$ exit and are positive definite.

The estimation procedure discussed above will be limited to the dynamic admissible parameter space abbreviated as $\operatorname{DAPS}(\delta, \omega)$.

The issue of how to measure volatility has generated series of ideas; various methods proposed include the moving standard deviation of percentage changes in exchange rate (Ait-Sahalia and Hansen, 2005) and the periodic volatility (Ederington and Guan, 2004). The generalised volatility $\sigma_{p}$ for $p$ used in this study is defined as Eq. 7

$\sigma_{\mathrm{p}}=\sigma_{\mathrm{z}} \mathrm{p}^{1 / 2}=\mathrm{h}(\mathrm{p}=\mathrm{t})$

where, $\sigma_{\mathrm{z}}^{2}$ is the variance of the outlier free time series, given that $\sigma^{2}$ is known, then Eq. 8:

$\mathrm{E}\left[\sigma_{\mathrm{p}}^{2}\right]=\sigma_{\mathrm{z}}^{2}$ pand $\operatorname{Var}\left(\sigma_{\mathrm{p}=\mathrm{t}}^{2}\right)=\mathrm{p}^{2} \operatorname{Var}\left(\sigma_{\mathrm{p}}^{2}\right)=\frac{2 \mathrm{p}^{2} \sigma_{\mathrm{p}}^{2}}{\mathrm{n}-1}$

We assume that $\mathrm{n}$ is large and the normal distribution test statistic can be used to test the significant of the volatility at time $\mathrm{t}$.

Estimation of auto covariance and parameters of outlier dynamic volatility model: We can rewrite Eq. (5), an outlier dynamic volatility model of order $p, q$ (DM (p, q)) as Eq. 9:

$\delta(B) Y_{t}=\omega(B)\left[Z_{t}^{\wedge}(t)\right]+e_{t}$

And define $Z_{t}^{\wedge}(t)=Z_{t}+D \xi_{t}^{T}$. Multiply Eq. 9 by $\mathrm{Y}_{\mathrm{t}-\mathrm{r}}, \mathrm{r}=1,2, \ldots \ldots \mathrm{K}$ and take expectation gives:

$\delta(B) E\left(Y_{t} Y_{t-r}\right)=\omega(B)\left[E\left(Z_{t}^{\wedge}(t) Y_{t-r}\right)\right]+E\left(e_{t} Y_{t-r}\right)$, but for all $r>1$ this expression reduces to: 
$\delta(\mathrm{B}) \gamma_{\mathrm{Y}}(\mathrm{r})=\omega(\mathrm{B})\left[\gamma_{\mathrm{YZ}^{\wedge}}(\mathrm{r})\right]$

Since $\delta_{i} B^{p}=\delta_{i-p}$ and $\omega_{j} B^{p}=\omega_{j-p}$, then by Eq. 10 we estimate the Auto Covariance Functions (ACF) of the output series as Eq. 11:

$$
\gamma_{Y}(r)=\sum_{i=0}^{p-1} \delta_{i} \gamma_{Y}(r)+\sum_{j=0}^{q} \omega_{j} \gamma_{Y Z^{\wedge}}(r)
$$

Clearly the ACF of dependent variable of the dynamic model defined in Eq. 5 is embedded with outlier volatility input.

To estimate the parameters of the dynamic model, we differentiate $\sum_{\mathrm{t}=1}^{\mathrm{n}} \mathrm{e}_{\mathrm{t}}^{2}$ with respect to $\delta(\mathrm{B})$ and $\omega(\mathrm{B})$ respectively give: $\frac{\mathrm{d}\left(\sum_{\mathrm{t}=1}^{\mathrm{n}} \mathrm{e}_{\mathrm{t}}^{2}\right)}{\partial(\delta(\mathrm{B}))}=2\left\{\sum_{\mathrm{t}=1}^{\mathrm{n}} \delta(\mathrm{B}) \mathrm{Y}_{\mathrm{t}}^{2}-\sum_{\mathrm{t}=1}^{\mathrm{n}} \omega(\mathrm{B}) \mathrm{Y}_{\mathrm{t}} \mathrm{Z}_{\mathrm{t}}^{\wedge}(\mathrm{t})\right\}$, that is Eq. 12:

$$
\frac{d\left(\sum_{t=1}^{n} e_{t}^{2}\right)}{\partial(\delta(B))}=\delta(B) \sum_{t=1}^{n} Y_{t}^{2}-\omega(B) \sum_{t=1}^{n} Y_{t} Z_{t}^{\wedge}(t)=0
$$

Also, $\frac{d\left(\sum_{t=1}^{n} e_{t}^{2}\right)}{\partial(\omega(B))}=2\left\{\sum_{t=1}^{n} \delta(B) Y_{t} Z_{t}^{\wedge}(t)-\sum_{t=1}^{n} \omega(B) Z_{t}^{2 \wedge}(t)\right\}$ and thus

$$
\frac{d\left(\sum_{t=1}^{n} e_{t}^{2}\right)}{\partial(\omega(B))}=\sum_{t=1}^{n} \delta(B) Y_{t} Z_{t}^{\wedge}(t)-\sum_{t=1}^{n} \omega(B) Z_{t}^{2 \wedge}(t)=0
$$

Solving the normal Eq. 12 and 13 and with appropriate starting values we can obtain the least squares estimates of $\mathrm{V}=(\delta, \omega)$.

Two major points of interest are noticeable in Eq. 11-13. In the first case, the magnitude of the outlier as well as the volatility in the input series have a bandwagon effect on the autocovariance generated in (11), according to Chernick et al. (1982), this may jeopardize the autocovariance function as an estimation and identification tool. Bartlett (1946) and Shangodoyin (2011) have claimed that where series $\left(Z_{t}, D_{t}, Y_{t}\right)$ are themselves auto correlated, the lagged cross-correlation estimates can have high variance and the estimate at different lags can be highly correlated with one another, this situation can be attributed to the presence of outlier in series and masking of the volatility. In the second case, the contribution of series $D_{t}$ and $h_{t}(t)$ on the estimate $\mathrm{V}=(\delta, \omega)$ is readily seen in the derivations from the normal Eq. 12 and 13. Finally the analysis of outlier in series may start with the elimination of volatility as this may be masked with $\mathrm{D}_{\mathrm{t}}$.
Variance of $\mathbf{R}_{\mathbf{t}}$ and test of adequacy: We have earlier defined the resident in the outlier DM volatility model in second part of Eq. 6 as $\mathrm{R}_{\mathrm{t}}$, where $R_{t}=\frac{\omega(B) D_{B} \xi_{t}^{T}}{\delta(B)}+\delta^{-1}(B) e_{t}=\theta(B) D_{t=T}+\delta^{-1}(B) e_{t}$.

Following $\mathrm{T}$ say (1986), the magnitude of the outlier based on the least squares theory is:

$$
\begin{aligned}
& D_{t}=\psi(B) P_{t}, \text { where } P_{t}=\theta(B) X_{t}= \\
& \frac{\omega(B)}{\delta(B)}=\left(1-\theta_{1} B-\ldots-\theta_{m} B^{m}-\ldots\right) X_{t}
\end{aligned}
$$

With $\quad \psi(\mathrm{B})=\left(1-\theta_{1} \mathrm{~B}-\theta_{2} \mathrm{~B}^{2}-\ldots \ldots . \theta_{\mathrm{m}} \mathrm{B}^{\mathrm{m}}\right)^{-1} \quad$ are obtained after fitting ARMA model to the outlier free series $h_{t}(t)$. By assumptions (ii) and (iii), the variance of $R_{t}$ is Eq. 14:

$\operatorname{Var}\left(\mathrm{R}_{\mathrm{t}}\right)=(\theta(\mathrm{B}))^{2} \sigma_{\mathrm{D}_{(\mathrm{t}=\mathrm{T})}}^{2}+\delta^{-2}(\mathrm{~B}) \sigma_{\mathrm{e}}^{2}$

From (13) it is evident that for an outlier contaminated series, the variability in the residual model is increased by $(\theta(\mathrm{B}))^{2} \sigma_{\mathrm{D}_{(\mathrm{t}=\mathrm{T})}}^{2}$ and the precision of the output generated can be measured through the variance of $\mathrm{R}_{\mathrm{t}}$. The residual cross-correlation $\rho_{\mathrm{k}}^{*}=\frac{\sum_{\mathrm{t}=1}^{\mathrm{n}} \mathrm{x}_{\mathrm{t}} \mathrm{e}_{\mathrm{t}}}{\left\{\sum_{1}^{\mathrm{n}} \mathrm{x}_{t}^{2} \sum_{1}^{n} \mathrm{e}_{t}^{2}\right\}^{\frac{1}{2}}}$ is employed in checking adequacy of fit, because large cross-correlation between $\left\{\mathrm{e}_{\mathrm{t}}\right\}$ and $\left\{\mathrm{X}_{\mathrm{t}}\right\}$ reveals inadequacy in the dynamic structure $\omega(\mathrm{B}) / \delta(\mathrm{B})$. The test criterion is the quantity $\chi^{2}=\mathrm{n} \sum_{\mathrm{k}=1}^{\mathrm{v}}\left(\rho_{\mathrm{k}}^{*}\right)^{2}$, which possesses a $\lambda^{2}$ distribution with $v-p-q$ degrees of freedom. The equation derived above will be examined empirically in this study under results and discussion. with some well analysed data using a SYSTAT statistical package.

We use as Series A data on Quarterly Nigerian Agricultural Export Crops between January, 2000 and December, 2010 as dependent variable taken along with the quarterly exchange rate over twelve years between 2000 and 2010. Series B is the quarterly sales of Botswana meat and meat product as dependent variable and average monthly exchange rate between quarter 1 , 1997 and quarter 42003.

\section{RESULTS AND DISCUSSION}

Empirical illustration of outlier with volatility: We evaluate the volatility input using Eqs. 7 and 8 by the normal distribution test statistic and whenever the volatility is insignificant we neglect it effects, otherwise we eliminate the value of volatility using $\mathrm{z}_{\mathrm{t}}-\mathrm{h}(\mathrm{t})$. 
To examine the contribution of volatility $h_{t}(t)$ series in DM model specification as well as its effects on output series generated; we assume an outlier input series and the timing of the outlier are observed as well as the volatility. The estimates of the dynamic structure are computed with their variances; the variance of the model residual is computed and the actual test of adequacy is performed. A comparison is made between DM model with or without outlier input series. We shall now analyses some series using the approach discussed.

Series A: This is an economic time series of size 44; we fitted the DM $(1,1)$ models $\mathrm{Y}_{\mathrm{t}}=0.999 \mathrm{Y}_{\mathrm{t}-1}+\mathrm{X}_{\mathrm{t}}+0.915$ $\mathrm{X}_{\mathrm{t}-1}+\mathrm{R}_{\mathrm{t}}$ and $\mathrm{Y}_{\mathrm{t}}=\mathrm{X}_{\mathrm{t}}+0.808 \mathrm{X}_{\mathrm{t}-1}+\mathrm{R}_{\mathrm{t}}$, to the series when the input is outlier free and contaminated respectively.

It evident from Table 1 that volatility in series A is significantly $\mathrm{p}$ [pronounced and series $\mathrm{B}$ does not suffer any significant volatility. We accordingly remove the volatility in series A and model the resultant series with and without outliers. The results are displayed in table 2.

Table 1: Significant test on Volatility

\begin{tabular}{lllll}
\hline Series data & T-statistics & $\mathrm{DF}=\mathrm{n}-2$ & Probability & Remark on ht \\
\hline A & 3.15 & 10 & 0.001 & Significant \\
B & 0.111 & 19 & 0.515 & Not Sig \\
\hline
\end{tabular}

Table 2: Estimates of the models fitted with their standard errors in bracket and value of the test criterion

\begin{tabular}{lllll}
\hline & $\begin{array}{l}\text { Series } \\
\text { A }\end{array}$ & $\begin{array}{l}\text { Series } \\
\mathrm{A}^{*}\end{array}$ & $\begin{array}{l}\text { Series } \\
\mathrm{B}\end{array}$ & $\begin{array}{l}\text { Series } \\
\mathrm{B}^{*}\end{array}$ \\
$\begin{array}{l}\text { Type of } \\
\text { series }\end{array}$ & $\begin{array}{l}\text { Without } \\
\text { Outlier }\end{array}$ & $\begin{array}{l}\text { Without } \\
\text { outlier }\end{array}$ & $\begin{array}{l}\text { With } \\
\text { outlier }\end{array}$ & $\begin{array}{l}\text { Without } \\
\text { outlier }\end{array}$ \\
\hline $\begin{array}{l}\text { Number } \\
\text { of outlier } \\
\text { Timing (T) }\end{array}$ & 2 & Nil & 5 & Nil \\
& 2,11 & - & $1,2,3,4$ & - \\
$\begin{array}{l}\delta \text {-Estimate } \\
\delta_{1}\end{array}$ & 0.679 & 0.489 & 0.533 & 0.538 \\
$\delta_{2}$ & $(0.115)$ & $(0.142)$ & $(0.187)$ & $(0.174)$ \\
& - & 0.265 & - & - \\
$\omega_{-}$Estimate & & $(0.144)$ & & \\
$\omega_{0}$ & 0.074 & 0.120 & 0.728 & 0.974 \\
$\omega_{1}$ & $(0.35)$ & $(0.013)$ & $(0.912)$ & $(0.867)$ \\
& 0.999 & 0.998 & 1.950 & 1.716 \\
& $(0.082)$ & $(0.112)$ & $(0.883)$ & $(0.869)$ \\
& 0.915 & 0.887 & & \\
$\begin{array}{l}\text { Variance } \\
\text { of } \mathrm{R}_{\mathrm{t}}\end{array}$ & $(0.010)$ & $(0.027)$ & & 1.063 \\
$\begin{array}{l}\text { Test } \\
\text { criterion } \mathrm{Q}^{*}\end{array}$ & 0.174 & 0.121 & 1.309 & .766 \\
Prob (Q)* & 4.459 & 3.662 & 7.832 & 4.611 \\
\hline & 0.521 & 0.462 & 0.00065 & 0.203 \\
\hline
\end{tabular}

In Table 2, the residual variance of the contaminated model is 1.359 multiple of that obtained for model are greater than that obtained for outlier contaminated model; but the test for the DM model with outlier is less when compared to what is obtained for outlier free model. The test statistics indicates that both models are adequate, but the power of the test for the model without outlier gives no room for questioning model adequacy more satisfactory than what is obtained for outlier contaminated model.

Series B: The series is different once before attaining stationary and we fitted the DM $(1,1,1)$ models: $\mathrm{Y}_{\mathrm{t}}=$ $0.533 \mathrm{Y}_{\mathrm{t}-1}+0.728 \mathrm{X}_{\mathrm{t}}+1.950 \mathrm{X}_{\mathrm{t}-1}+\mathrm{R}_{\mathrm{t}}$ and $\mathrm{Y}_{\mathrm{t}}=538 \mathrm{Y}_{\mathrm{t}-1}$ $+0.924 \mathrm{X}_{\mathrm{t}}+1.716 \mathrm{X}_{\mathrm{t}-1}+\mathrm{R}_{\mathrm{t}}$ to the series $\left(\mathrm{Y}_{\mathrm{t}}, \mathrm{X}_{\mathrm{t}}\right)$ when the input is with and without respectively. In Table 2, the model residual variance with outlier is 1.231 multiple of the model residual variance without outlier. The magnitude of the standard error of the estimates of $\delta_{1}, \omega_{0}$ and $\omega_{1}$ are smaller for outlier free model than what obtained for outlier contaminated model, but the estimate of $\omega_{0}$ for outlier free model is not significant. The test statistics rejects model fitted on outlier contaminated series as adequate, where as the power of test model without outlier gives no room for questioning model adequacy.

\section{CONCLUSION}

The study made use of a statistical technique which considers the time series prosperities of the variables involved. This eliminates the possibility of spurious regressions and consequently avoids inefficient estimators.

The analysis of outlier in DM modelling should involve the determination of volatility, most especially in economic series suitable for use. The model described in Eq.3 through 13 has a lot to recommend it on the contributions of outlier embedded with volatility in empirical study on dynamic function modelling. In the first instance, outlier significantly affects the estimates of the model, apart from this; the model residual is affected, these will have a combine effect on the precision of output generated. Secondly, the test criterion is jeopardized as tool for measuring adequacy of fit; because outlier series are embedded in its computation.

\section{REFERENCES}

Ait-Sahalia, Y. and L. Hansen, 2005. Handbook of Financial Econometrics Set. 1st Edn., Elsevier Science and Technology, Boston, ISBN-10: 0444535543, pp: 1000.

Bartlett, M.S., 1946. On the theoretical specification and sampling properties of autocorrelated timeseries. Supplement J. Royal Stat. Soci., 8: 27-41. 
Chernick, M.R., D.J. Downing and D.H. Pike, 1982. Detecting outliers in time series data. J. Am. Stat. Asso., 77: 743-747.

Ederington, L.H. and W. Guan, 2004. Forecasting Volatility. Social Science Electronic Publishing, Inc.

Rapsomanikis, G. and S. Alexander, 2006. The Impact of Domestic and International Commodity Price Volatility on Agricultural Income Instability: Ghana, Vietnam and Peru. 1st Edn., United Nations University, Helsinki, ISBN: 9291908320, pp: 21.

Sarnaglia, A.J.Q., V.A. Reisen and C. Levy-Leduc, 2010. Robust estimation of periodic autoregressive processes in the presence of additive outliers. J. Multivariate Anal. 101: 2168-2183. DOI:10.1016/J.JMVA.2010.05.006
Shangodoyin, D.K., 2008. On the selection of best subset autoregressive models in prediction with application to real life data. Statistics Transition. 9: 471-484

Shangodoyin, D.K., 2011. Identification of transfer function model in the presence of aberrant leading indicator. University of Botswana.

Tsay, R.S., 1986. Time series model specification in the presence of outliers. J. Am. Stat. Asso., 81: 132-141. 